\title{
COMPASS results on gluon polarisation
}

\author{
Luis SILVA ${ }^{* \dagger}$ \\ LIP Lisbon \\ E-mail: lsilva@lip.pt
}

One of the missing keys in the present understanding of the spin structure of the nucleon is the contribution from the gluons: the so-called gluon polarisation. This quantity can be determined in DIS through the Photon-Gluon Fusion (PGF) process, in which two analysis methods may be used: (i) identifying open charm events or (ii) selecting events with high- $p_{T}$ hadrons. The data used in the present work were collected by the COMPASS Experiment, where a naturally polarised muon beam of $160 \mathrm{GeV}$, impinging on a polarised nucleon fixed target, is used. Preliminary results for the gluon polarisation from high- $p_{T}$ and open charm analyses are presented. The gluon polarisation result for high- $p_{T}$ hadrons is divided, for the first time, into 3 statistically independent bins at LO. The result from open charm analysis is obtained at LO and NLO. In both analyses a new weighted method based on a neural network approach is used.

The 2011 Europhysics Conference on High Energy Physics-HEP 2011,

July 21-27, 2011

Grenoble, Rhône-Alpes France

\footnotetext{
* Speaker.

'On behalf of the COMPASs Collaboration.
} 


\section{Introduction}

The nucleon spin sum rule can be written in a heuristic way as: $\frac{1}{2}=\frac{1}{2} \Delta \Sigma+\Delta G+L_{q, g}$, where $\Delta \Sigma$ and $\Delta G$ are the quark and gluon contributions to the nucleon spin, respectively, and $L_{q, g}$ is the parton orbital angular momentum. In the late 80's it was announced and confirmed from several experiments that the contribution carried by the quarks is $\sim 1 / 3$ of the nucleon spin. The purpose of this work is to estimate the gluon polarisation $\Delta G / G$, which is deeply related with the gluon contribution to the nucleon spin.

\section{Gluon Polarisation Measurements}

The spin dependent effects are measured experimentally using the helicity asymmetry $A_{\mathrm{LL}}^{\text {exp }}$ defined as $\frac{\sigma^{\rightleftarrows}-\sigma^{\leftrightarrows}}{\sigma^{\rightleftarrows}+\sigma^{\leftrightarrows}}$ where $(\leftleftarrows)$ and $(\leftrightarrows)$ refer to the parallel and anti-parallel spin helicity configuration of the beam lepton $(\leftarrow)$ with respect to the target nucleon $(\Leftarrow$ or $\Rightarrow)$. The data for the present analyses was taken in the COMPASs experiment [1]. The gluon polarisation can be measured via the Photon-Gluon Fusion (PGF) process, depicted in fig. $1 \mathrm{c}$ ), which allows to probe the spin of the gluon inside the nucleon. The PGF process may be selected using two analysis methods: (i) selecting high $p_{T}$ hadron events, or (ii) selecting events containing open charm mesons.

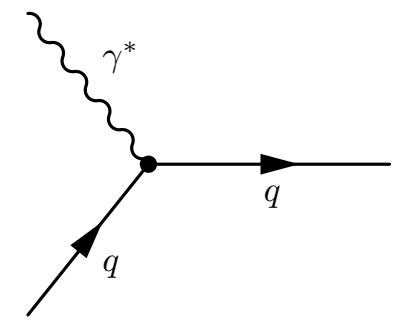

a)

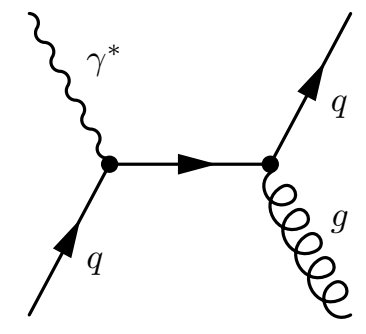

b)

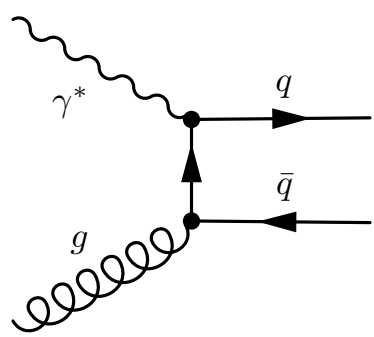

c)

Figure 1: DIS Feynman diagrams for $\gamma^{\star} N$ scattering: a) virtual photo-absorption (LP), b) gluon radiation (QCD Compton) and c) photon-gluon fusion (PGF).

In the high $p_{T}$ analysis the spin helicity asymmetry is calculated by selecting events containing high $p_{T}$ hadron pairs above 0.7 and $0.4 \mathrm{GeV} / c$, respectively for the highest and the second highest $p_{T}$ hadron with respect the virtual photon direction. A cut on $Q^{2}>1(\mathrm{GeV} / c)^{2}$ was also applied in order to select DIS events. Two other processes compete with the PGF process in LO QCD approximation, namely the virtual photo-absorption leading order process (LP) and the gluon radiation (QCD Compton) process, illustrated in fig. 1. The spin helicity asymmetry for the high $p_{T}$ hadron pair data sample can thus be schematically written as:

$$
A_{\mathrm{LL}}^{2 h}\left(x_{B j}\right)=R_{\mathrm{PGF}} a_{\mathrm{LL}}^{\mathrm{PGF}} \frac{\Delta G}{G}\left(x_{G}\right)+R_{\mathrm{LP}} D A_{1}^{\mathrm{LO}}\left(x_{B j}\right)+R_{\mathrm{QCDC}} a_{\mathrm{LL}}^{\mathrm{QCDC}} A_{1}^{\mathrm{LO}}\left(x_{C}\right) .
$$

The process fractions are represented by $R_{i}, i$ referring to the different processes. $a_{\mathrm{LL}}^{i}$ represents the partonic cross section asymmetries, $\Delta \hat{\sigma}^{i} / \hat{\sigma}^{i}$, also known as analysing power. The depolarisation factor $D$ is the fraction of the muon beam polarisation transferred to the virtual photon. $A_{1}^{\mathrm{LO}}$ is the virtual photon asymmetry. A similar eq. to (2.1) can be written to express the inclusive asymmetry 
of a data sample, $A_{\mathrm{LL}}^{i n c l}$. Using eq. (2.1) for the high $p_{T}$ hadron pair sample and the same equation but for an inclusive sample, the final expression to extract the gluon polarisation is obtained:

$$
\frac{\Delta G}{G}\left(x_{G}^{a v}\right)=\frac{A_{\mathrm{LL}}^{2 h}\left(x_{B j}\right)+A^{\text {corr }}}{\lambda} .
$$

This formula corresponds to the spin helicity asymmetry $A_{\mathrm{LL}}^{2 h}$, measured directly from data, plus a correcting asymmetry $A^{\text {corr }}$ involving mainly other two processes. The $\lambda$ factor relates the partonic asymmetries and the fractions of the involved processes. The partonic asymmetries and the process fractions need to be estimated using a dedicated and well tuned Monte Carlo (MC) simulation. A bayesian Neural Network (NN) [2] approach was used. The purpose of such approach is to assign an event probability for each process involved, represented by the process fractions in eq. (2.1). The $\mathrm{NN}$ is also used to provide the partonic asymmetries and $x_{C}$ and $x_{G}$ variables. Details about the high $p_{T}$ analysis, for $Q^{2}>1(\mathrm{GeV} / c)^{2}$, can be found in [3]. A similar analysis was performed for the $Q^{2}<1(\mathrm{GeV} / c)^{2}$ data. This separation is due to the physical processes contained in the two $Q^{2}$ regimes. The $Q^{2}<1(\mathrm{GeV} / c)^{2}$ regime represents the quasi-real photon, in such conditions the photon may exhibit some inner structure. Therefore beside the three already mentioned processes the photon structure processes need to be included in the MC simulation. Details of this analysis can be found in [4].

For the open charm analysis the spin helicity asymmetries are calculated using a data with $D^{0}$ mesons in the final state. These events are selected from their decaying products, i.e. $K \pi$ pairs. To achieve this selection a good particle identification is required. Applying a set of kinematic cuts the combinatorial background originated from processes in which the virtual photon strikes a parton inside the nucleon is reduced. In addition the background is even more suppressed by tagging the $D^{0}$ candidates with a parent $D^{\star}$ using the $D^{\star} \rightarrow D^{0} \pi_{\text {slow }}$ channel. In this way three additional channels were included in the final analysis, $D^{0}$ to $K_{\text {sub }} \pi, K \pi \pi^{0}$ and $K \pi \pi \pi$. The former includes kaon candidates not identified by the RICH detector [1].

The number of events with $D^{0}$ particles in the final state is related to the gluon polarisation as shown by this expression:

$$
N_{t}=\alpha(S+B)\left[1+\beta\left(a_{\mathrm{LL}} \frac{S}{S+B} \frac{\Delta G}{G}+D \frac{B}{S+B} A^{\mathrm{bg}}\right)\right]
$$

The subscript $t$ on the number of events corresponds to the possible muon target spin configurations. The $\alpha$ factor contains the acceptance, muon flux and number of nucleons and $\beta$ the beam and target polarisations and dilution factor. $S$ and $B$ represent the number of signal and background events taken under the invariant mass spectrum peak. $\frac{S(B)}{S+B}$ is the signal (background) purity. $A^{\text {bg }}$ is the asymmetry of the combinatorial background. Taking into account all the possible muon target spin configurations a set of equations is derived from eq. (2.3). Still, to solve this system of equations the partonic asymmetry $a_{\mathrm{LL}}$ and the signal purity $\frac{S}{S+B}$ must be estimated. To compute the partonic asymmetry $a_{\mathrm{LL}}$ a dedicated MC simulation is used. A NN approach is designed to parametrise the partonic asymmetry and the signal significance $\frac{S}{S+B}$. Details about this analysis can be found in [5]. A NLO QCD analysis was also performed. Into the analysing power NLO QCD virtual and gluon bremstrahlung corrections were included to the PGF process, as well as background processes. 


\section{Results}

The preliminary results on gluon polarisation using the high $p_{T}\left(Q^{2}<1\right.$ and $Q^{2}>1(\mathrm{GeV} / c)^{2}$, LO QCD order) and open charm (LO and NLO QCD order) analyses are now presented. The $\Delta G / G$ value obtained in high $p_{T}$ analysis, for $Q^{2}>1(\mathrm{GeV} / c)^{2}$, averaged at $x_{g}=0.09_{-0.04}^{+0.08}$ was found to be equal to $\Delta G / G=0.125 \pm 0.060_{\text {stat }} \pm 0.063_{\text {sys. }}$. This measurement is presented in three statistically independent points, in 1 . The same result for $Q^{2}<1(\mathrm{GeV} / c)^{2}$ is $\Delta G / G=0.016 \pm$ $0.058_{\text {stat }} \pm 0.055_{\text {sys }}$.

\begin{tabular}{llll}
\hline & $1^{\text {st }}$ Bin & $2^{\text {nd }}$ Bin & $3^{\text {rd }}$ Bin \\
\hline$\Delta G / G$ & $0.147 \pm 0.091 \pm 0.088$ & $0.079 \pm 0.096 \pm 0.082$ & $0.185 \pm 0.165 \pm 0.143$ \\
$x_{G}^{a v}$ & $0.07_{-0.03}^{+0.05}$ & $0.10_{-0.04}^{+0.07}$ & $0.17_{-0.06}^{+0.10}$ \\
\hline
\end{tabular}

Table 1: Gluon polarisation results in bins of $x_{G}$.

The gluon polarisation value for the open charm analysis was found as $\Delta G / G=-0.08 \pm$ $0.21_{\text {stat }} \pm 0.08_{\text {sys }}$ averaged at $x_{G}=0.11_{-0.05}^{+0.11}$ for LO QCD order and $\Delta G / G_{N L O}=-0.20 \pm 0.21_{\text {stat }} \pm$ $0.08_{\text {sys }}$ averaged at $x_{G}=0.28_{-0.10}^{+0.19}$ at NLO QCD order. The gluon polarisation values from high $p_{T}$ hadrons are measured at a hard scale of $\left\langle\mu^{2}\right\rangle=3.4(\mathrm{GeV} / c)^{2}$, while the ones from open charm mesons were evaluated at a hard scale of $\left\langle\mu^{2}\right\rangle=13(\mathrm{GeV} / c)^{2}$. All the gluon polarisation results of the COMPASS Collaboration are summarised in fig. 2 together with the SMC [6] and HERMES [7] results.
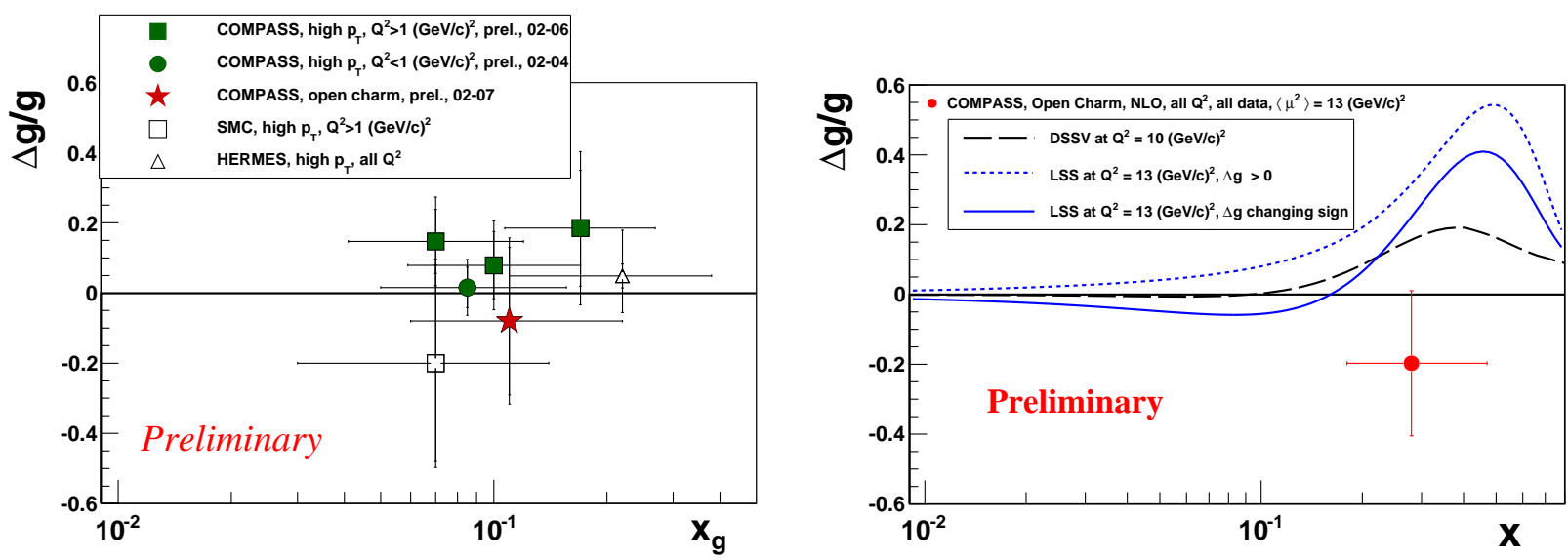

Figure 2: Gluon polarisation results: LO QCD order results (left side plot), NLO QCD order results and also theorical fits form the DSSV [8] and LSS [9] (right side plot).

\section{Conclusions}

The gluon polarisation preliminary results presented here show that, in the region of $x_{g} \simeq 0.1$, these results are compatible with all the remain ones from other analyses, and also compatible with a small value for $\Delta G / G$. This result is also confirmed by the NLO QCD global fit curves in fig. 2 (right side plot) which also predicts a small value for the gluon contribution to the nucleon spin. 


\section{References}

[1] Abbon B, et al. 2007 Nucl. Instrum. Meth. A 577 455-518 (Preprint hep-ex/0703049)

[2] Sulej R, Zaremba K, Kurek K and Rondio E 2007 Measur. Sci. Tech. 18 2486-90

[3] Silva L and the COMPASS Collaboration 2011 J. Phys.: Conf. Ser. 295012067

[4] Ageev E, et al. 2006 Phys. Lett. B 633 25-32 (Preprint hep-ex/0511028)

[5] Franco C and the COMPASS Collaboration 2010 Proc. of Science DIS2010 225

[6] Adeva B, et al. 2004 Phys. Rev. D 70012002

[7] Airapetian A, et al. 2000 Phys. Rev. Lett. 84 2584-8

[8] Leader E, Sidorov A and Stamenov D 2010 Phys. Rev. D 82 114018-29

[9] de Florian D, Sassot R, Stratmann M and Vogelsang W 2009 Phys. Rev. D 80 034030-55 